\title{
A decentralised model of psychiatric care: Profile, length of stay and outcome of mental healthcare users admitted to a district-level public hospital in the Western Cape
}

\author{
E Thomas, ${ }^{1} \mathrm{MB}$ ChB, Dip HIV Mx; K J Cloete, ${ }^{1} \mathrm{PhD} ; \mathbf{M}$ Kidd, ${ }^{2} \mathrm{PhD} ;$ H Lategan, ${ }^{1}$ MB ChB, FCPsych, MMed (Psych) \\ ${ }^{1}$ Department of Psychiatry, Faculty of Medicine and Health Sciences, Stellenbosch University, Cape Town, South Africa \\ ${ }^{2}$ Centre for Statistical Consultation, Stellenbosch University, Cape Town, South Africa
}

Corresponding author: E Thomas (lene_tnt@live.co.za)

\begin{abstract}
Background. There is a lack of studies assessing the profile and outcome of psychiatric patients at entry-level public hospitals that are prescribed by the Mental Health Care Act to provide a decentralised model of psychiatric care.

Objective. To assess the demographic and clinical profile as well as length of stay and outcomes of mental healthcare users admitted to a district-level public hospital in the Western Cape.

Method. Demographic data, clinical diagnosis, length of stay, referral profile and outcomes of patients $(N=487)$ admitted to Helderberg Hospital during the period 1 January 2011 - 31 December 2011 were collected.

Results. Psychotic disorders were the most prevalent ( $n=287,59 \%)$ diagnoses, while 228 (47\%) of admission episodes had comorbid/ secondary diagnoses. Substance use disorders were present in 184 (38\%) of admission episodes, 37 (57\%) of readmissions and 19 (61\%) of abscondments. Most admission episodes $(n=372,76 \%)$ were discharged without referral to specialist/tertiary care.

Conclusion. Methamphetamine use places a significant burden on the provision of mental healthcare services at entry-level care. Recommendations for improving service delivery at this district-level public hospital are provided.
\end{abstract}

S Afr J Psychiatr 2015;21(1):8-12. DOI:10.7196/SAJP.538

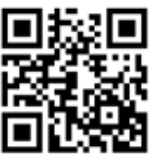

In South Africa (SA), the Western Cape Province has the highest provincial 12-month and lifetime prevalence of mental disorders. ${ }^{[1]}$ Mental disorders occur here in $25 \%$ of adults and $17 \%$ of children and adolescents. ${ }^{[2]}$ The burden of mental disorders in this province is further compounded by the increasing trend in substance abuse, ${ }^{[3]}$ which places an increased demand on psychiatric inpatient services for use of sedation, seclusion and length of stay. ${ }^{[4]}$

To bring SA in line with international mental health legislation initiatives and trends, the SA Mental Health Care Act No. 17 (MHCA) was promulgated in 2002. ${ }^{[5]}$ The Act seeks to deinstitutionalise and shift the system from a custodial approach to one that encourages rehabilitative, community-based care, in order to ensure that psychiatric patients receive the best possible care, treatment and rehabilitation at all levels of healthcare. To increase accessibility to primary healthcare for psychiatric patients, selected regional and district public hospitals were prescribed to undertake a 72-hour observation period on mental healthcare users, ${ }^{[5]}$ to allow a patient to be admitted, assessed every 24 hours and treated for 72 hours, without his or her consent, in order to: (i) exclude possible organic pathology; (ii) allow adequate treatment and management of possible substance-induced, medically related psychiatric disturbances or behavioural problems; and (iii) allow patients who may recover suitably during this 72 -hour period to gain good insight and judgement to either consent to further treatment or be discharged within 72 hours. ${ }^{[6]}$ After the 72 -hour observation period, two mental healthcare professionals reassess the patient and submit the recorded examination and findings to the head of the health establishment, who decides whether further treatment is required at a designated specialist psychiatric hospital.

Helderberg Hospital in the Western Cape is a 120-bed, level-1 district hospital identified by the MHCA to admit, observe and treat mental healthcare patients for 72 hours prior to admission to a psychiatric hospital. The hospital serves the Helderberg basin and surrounding areas in the Overberg district. Mental healthcare users are assessed in the casualty department by a doctor with a Bachelor's degree in medicine (MB ChB), and if admission is required, are transferred to a general overnight ward. Patients remain in the general overnight ward for the duration of the admission period. The services of a dedicated mental healthcare nurse, intern doctor/medical officer/family physician registrar, shared family physician, and a visiting district psychiatrist are available, while patients also have access to an occupational therapist, physiotherapist, social worker, clinical psychologist and pastoral counsellor. There are no designated psychiatric beds nor a seclusion unit.

The increasing numbers of mental healthcare users at Helderberg Hospital and long waiting lists at specialist psychiatric hospitals have resulted in earlier discharges with outpatient follow-up. However, the hospital is not unique in the challenges it faces in providing the extent and scope of practices required as a designated 72-hour assessment unit. A review by Ramlall et al. ${ }^{[7]}$ on the effect of the MHCA on 49 designated regional and district hospitals in KwaZulu-Natal Province also highlights the lack of sufficient beds to accommodate 
the increasing clinical demand and the scarcity of seclusion rooms to manage acutely disturbed psychiatric patients within a general hospital setting. Moreover, the implementation of a decentralised model of psychiatric care has resulted in an increased burden on not only district-level, but also secondary-level and regional hospitals. Several studies accordingly highlight inadequate existing infrastructure and resources to meet the increasing need for mental healthcare. ${ }^{[2,7-9]}$ There is, however, a lack of data concerning the profile of mental healthcare users at entry level, which fuels deficits and inadequacies in service planning, as infrastructure and human resource allocation are planned according to hypothetical rather than real situations.

\section{Objectives}

The primary objective of this study was to assess the demographic and clinical profile, as well as length of stay and admission outcomes, of mental healthcare users receiving inpatient care at Helderberg Hospital over a 12-month period. Specific objectives included assessing:

- diagnostic profiles of mental healthcare users stratified by age and gender

- the percentage of admissions ascribed to substance abuse, particularly methamphetamine ('tik'), stratified by gender and age

- the length of stay prior to transfer to the next level of care, specifically to level-2 and -3 psychiatric hospitals or other clinical departments

- the length of stay as a function of diagnosis and demographic data

- rates of abscondments, discharges, readmissions, deaths, referrals and transfers to the next level of care (in accordance with the MHCA).

The increased demand on psychiatric inpatient services in the Western Cape and the lack of data on the profile of mental healthcare users, which should guide service planning at district level, underscore the current research.

\section{Method}

\section{Design, setting and participants}

This retrospective audit included all mental healthcare users admitted for a 72-hour assessment period (in accordance with the MHCA) to Helderberg Hospital, Somerset West, SA, between 1 January 2011 and 31 December 2011. Data on all mental healthcare users admitted were included, regardless of age. Information was extracted from patient folders. The sample size of 487 admission episodes reflects the number of new admissions and readmissions.

\section{Data collection and management}

Demographic data (age, gender), clinical diagnosis, length of stay (including data from those who were not discharged during the study period), referral pattern to the next level of psychiatric care or other clinical departments, and outcomes of admission (abscondment, discharge, readmission, transfer or death) were collected by a mental healthcare nurse and captured on a structured datasheet. A clinical diagnosis was made according to the following syndromal diagnostic category groupings: psychotic disorders (schizophrenia/other psychotic disorders/substance induced); substance use disorders, abuse and dependence (methamphetamine/cannabis/alcohol/other substances); mood disorders (bipolar mood disorder/major depressive disorder/ other mood disorder); anxiety disorders; dementias; suicide attempts; medical/surgical conditions; and other psychiatric diagnoses. While provisional admission diagnoses were made using the Diagnostic and Statistical Manual of Mental Disorders, 4th edition (DSM-IV) criteria, ${ }^{[10]}$ diagnoses were not verified in all instances by a psychiatrist or a psychiatric medical officer prior to transfer or discharge. Where a definitive diagnosis could not be provided, the foremost symptom or abnormal finding was recorded. A diagnosis of concurrent substance abuse was made on either the presenting clinical features, self-report by the patient, collateral history obtained or urinalysis.

A medical officer entered the data into an Excel spreadsheet, while the primary investigator reviewed, collated and validated the data. Missing data were extracted from either Clinicom or patient folders.

\section{Data analyses}

Data analyses were performed using Statistica version 10 (StatSoft Inc, USA). Descriptive statistics were reported as means and standard deviations (SDs) for ordinal data (e.g. age and length of stay) and frequencies as percentages of diagnoses. One-way analysis of variance (ANOVA) tests were used to compare ordinal data between groups, while cross-tabulations with the $\chi^{2}$ test were used to compare categorical variables. Spearman correlations were used to test for associations between ordinal variables. Significance was set at $p<0.05$.

\section{Ethics}

This study was approved by the Health Research Ethics Committee of Stellenbosch University (Reference number S12/06/180) and conducted in accordance with the International Committee for Harmonisation Good Clinical Practice (GCP) guidelines and SA GCP, as well as the Declaration of Helsinki and subsequent revisions. ${ }^{[1]]}$ Permission to use clinical data was obtained from the Provincial Government of the Western Cape and the medical superintendent of Helderberg Hospital. Anonymity was ensured by assigning a number to each participant.

\section{Results}

\section{Demographic data}

A total number of 487 psychiatric admission episodes were recorded for the period 1 January 2011 - 31 December 2011. The mean (SD) age of participants was 34 (14) years (range 12 - 86). Most patients were male $(n=270,55 \%)$.

\section{Clinical data}

Of the admission episodes, 245 (51\%) (Table 1) had comorbid/ secondary diagnoses. Psychotic disorders were the most frequently recorded clinical diagnostic cluster $(n=287,59 \%)$. Within this cluster, rates of mental and behavioural disorders due to psychoactive substance use $(n=162,56 \%)$, schizophrenia $(n=99,34 \%)$ and other psychotic disorders $(n=26,10 \%)$ were highest. Psychotic disorders were followed by substance use disorders $(n=184,38 \%)$, with methamphetamine $(n=88,70 \%)$, alcohol $(n=26,14 \%)$ and cannabis $(n=22$, $11 \%$ ) as the most frequently abused substances. Mood disorders formed the third most common clinical diagnostic cluster $(n=142,29 \%)$, with major depressive disorders highest $(n=81,58 \%)$ in this cluster.

\section{Clinical diagnoses and demographic data}

While no significant differences were found between the male and female mental health care users (MHCUs) diagnosed with psychotic 
Table 1. Psychiatric diagnoses by gender for mental healthcare users (total admission episodes, $N=487^{\star}$ ) admitted to Helderberg Hospital, 2011

\begin{tabular}{|c|c|c|c|c|}
\hline Diagnostic cluster & Total, $n(\%)$ & Males, $n(\%)$ & Females, $n(\%)$ & $p$-value ${ }^{\dagger}$ \\
\hline Psychotic disorders & $287(59)$ & $167(58)$ & $120(42)$ & 0.14410 \\
\hline $\begin{array}{l}\text { Substance-induced psychotic } \\
\text { disorder }\end{array}$ & & $111(69)$ & $51(31)$ & 0.00010 \\
\hline Schizophrenia & & $40(40)$ & $59(60)$ & \\
\hline Other psychotic disorder & & $16(62)$ & $10(38)$ & \\
\hline Mood disorders & $142(29)$ & $57(40)$ & $85(60)$ & 0.00001 \\
\hline Major depressive disorder & & $37(46)$ & $44(53)$ & \\
\hline Bipolar mood disorder & & $20(33)$ & $40(67)$ & \\
\hline Other mood disorders & & $0(0)$ & $1(100)$ & \\
\hline $\begin{array}{l}\text { Substance-use disorders (abuse, } \\
\text { dependence) }\end{array}$ & $184(38)$ & $121(66)$ & $63(34)$ & 0.00001 \\
\hline Methamphetamine (tik) & & $88(68)$ & $41(32)$ & 0.00001 \\
\hline Alcohol & & $16(9)$ & $10(5)$ & \\
\hline Cannabis & & $15(8)$ & $7(4)$ & \\
\hline Other substances & & $2(1)$ & $5(3)$ & \\
\hline Anxiety disorders & $6(1)$ & $3(50)$ & $3(50)$ & 0.78 \\
\hline Dementia & $16(3)$ & $8(50)$ & $8(50)$ & 0.66 \\
\hline Suicide attempts & $43(9)$ & $18(42)$ & $25(58)$ & 0.06 \\
\hline Intellectual disability & $6(1)$ & $5(83)$ & $1(17)$ & 0.14 \\
\hline Medical/surgical & $27(6)$ & $14(52)$ & $13(48)$ & 0.70 \\
\hline Other psychiatric disorders & $4(1)$ & $0(0)$ & $4(100)$ & 0.45 \\
\hline
\end{tabular}

disorders $(n=167 \quad(58 \%)$ v. $n=120 \quad(42 \%)$, respectively; $p=0.1441)$, differences were shown between males and females diagnosed with substance-induced psychotic disorder ( $n=111(69 \%)$ v. $n=51$ (31\%), respectively). Within the substance use disorder cluster, differences were also shown between males and females $(n=121(66 \%)$ v. $n=63(34 \%)$, respectively; $p=0.00001)$. Similarly, significantly more males $(n=88,68 \%)$ than females $(n=41,32 \%)$ used methamphetamine ( $p=0.00001$ ) (Table 1$)$. However, significantly more females $(n=85,60 \%)$ than males $(n=57$, $40 \%)$ were diagnosed with a mood disorder $(p<0.00001)$. Psychiatric diagnoses and methamphetamine-related admission episodes were most common in the 21 - 30 age group.

Length of stay as a function of outcome, diagnoses and demographic data

The mean (SD) length of stay was 5.4 (5.1) days. The mean length of stay was significantly longer in those who were transferred (7.7 (6.0) days) than those who were not transferred (4.7 (4.6) days) $(p<0.01)$.
Furthermore, the mean length of stay was significantly longer in those transferred to a psychiatric hospital (including Alexandra, Lentegeur, Stikland and Tygerberg) (9.4 (5.7) days) than those who were not transferred (4.7 (4.5) days) $(p<0.01)$.

The median length of stay prior to transfer to Tygerberg was 14 days, to Stikland 10 days, to Lentegeur 9 days, and to Alexandra 6 days. The queuing time (length of stay from date of admission to date of transfer, both dates inclusive) also varied significantly $(p<0.01)$ between the four specialist/tertiary referral hospitals (Fig. 1). These long waiting times may be due to the limited availability of beds for special population groups served by these centres, such as children and adolescents (Tygerberg Hospital) and the elderly (Stikland Psychiatric Hospital).

The main diagnostic grouping with the longest length of stay was dementia (median 7 days), followed by mood disorders (median 4 days), and substance-related disorders (median 4 days). A significant association was found between length of stay and age
Table 2. Outcome data of mental healthcare users (total admission episodes, $N=487$ ) admitted to Helderberg Hospital in 2011

\begin{tabular}{ll}
\hline & $\boldsymbol{n}(\%)$ \\
\hline Transferred & \\
No & $372(76)$ \\
Yes & $115(24)$ \\
Transferred to & \\
Lentegeur & $71(15)$ \\
Medical/surgical & $24(5)$ \\
Stikland & $11(2)$ \\
Tygerberg & $3(1)$ \\
Alexandria & $3(1)$ \\
Other facilities (medical/surgical) & $3(1)$ \\
Abscond & \\
No & $456(94)$ \\
Yes & $31(6)$ \\
Substance-related abscondments & $19(61)$ \\
Readmissions & $65(13)$ \\
Substance-related readmissions & $37(57)$ \\
Period before readmission & \\
(months) & \\
0 - 3 & $42(65)$ \\
$>3-6$ & $11(17)$ \\
$>6$ - 9 & $9(14)$ \\
$>99-12$ & $3(0.5)$ \\
&
\end{tabular}

(Spearman $r=0.09, p<0.05$ ), while there was no association with gender.

\section{Outcome data}

Of the 487 admission episodes recorded, $372(76 \%)$ entries received treatment, care and rehabilitation at Helderberg Hospital and were subsequently discharged home (Table 2). Of those transferred $(n=115,24 \%)$, 88 (18\%) were transferred to specialist/ tertiary psychiatric hospitals (Alexandra, Lentegeur, Stikland or Tygerberg), of which most $(n=71,15 \%)$ were transferred to Lentegeur Psychiatric Hospital. Admission episodes transferred to medical/surgical services amounted to 24 (5\%).

No deaths were recorded, while there were 31 (6\%) abscondments. Of the abscondments, 19 (61\%) were substance-related admissions, while $8(26 \%)$ were readmitted to the same facility. There were $65(13 \%)$ readmissions, and most $(n=42,65 \%)$ occurred within 3 months of discharge. Of the readmissions, 37 (57\%) were related to substance use either as primary or concomitant diagnoses. 


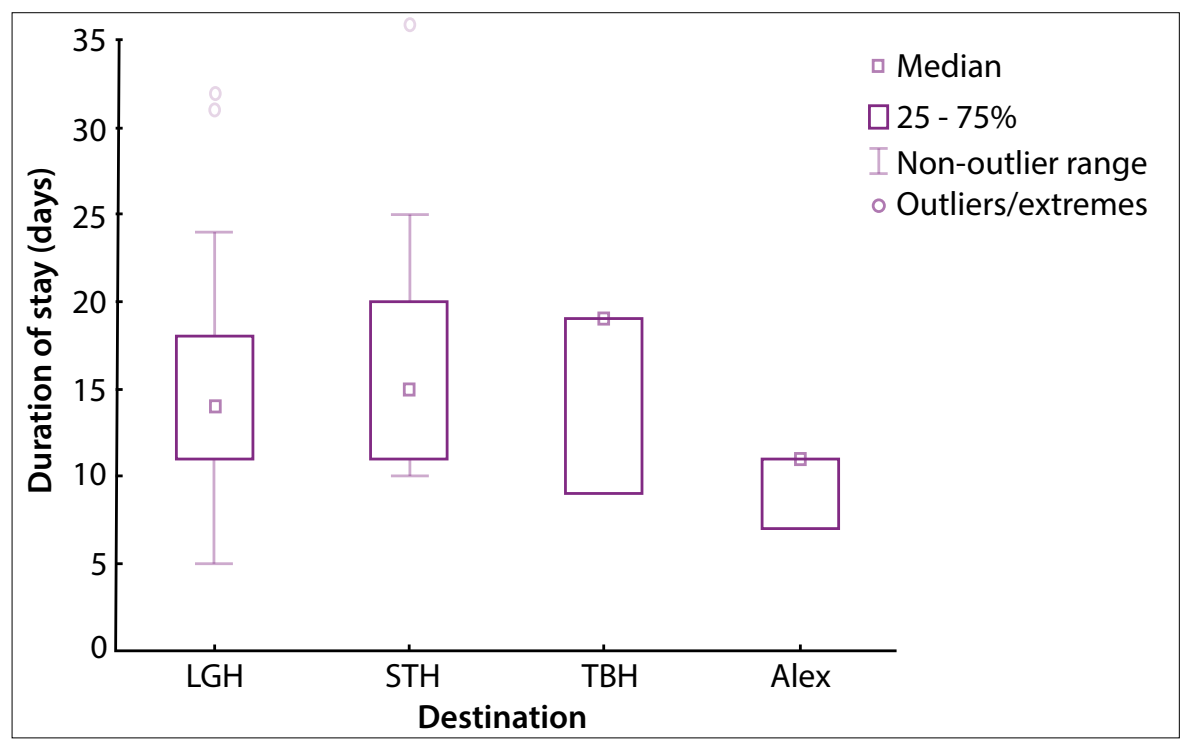

Fig. 1. Box and whisker plot showing duration of stay at the specialist psychiatric hospitals. (LGH = Lentegeur Hospital; $S T H=$ Stikland Hospital; $T B H=$ Tygerberg Hospital; Alex = Alexandria .)

\section{Discussion}

There is a lack of data on the profile of mental healthcare users at the entry level of healthcare, which could affect planning and provision of service delivery in the tiered system of mental healthcare. Therefore, the objective of this study was to assess the demographic and clinical profile as well as length of stay and admission outcomes of mental healthcare users presenting for treatment at a level-1 district hospital in the Western Cape, over a 12-month period. Demographic data showed that the average age of patients admitted was 34 years and that most were males, while clinical data showed that psychosis was most frequently recorded, and usually related to substance abuse, specifically of methamphetamine. Of further note is that $47 \%$ of the admission episodes had provisional secondary or comorbid diagnoses recorded. Of these, $42 \%$ were related to substance use; this group also accounted for $57 \%$ of readmissions. In terms of outcome measures, patients with dementia had the longest period of stay. Overall, 88 admission episodes (18\% of total) were transferred to specialist/tertiary psychiatric hospitals. The queuing time varied significantly between the specialist/ tertiary psychiatric hospitals, while those who were transferred to a specialist/tertiary psychiatric hospital stayed significantly longer at entry-level care.

The demographic and clinical profile of mental healthcare users presenting for treat- ment at Helderberg Hospital reflected findings from other SA studies on mental healththe Northern Cape Province on psychiatric patients similarly showed that most patients were male (65.8\%), with an average age of 32 years and diagnosed with schizophrenia (57.8\%) and substance-related disorders $(26.6 \%) .^{[12]}$ Another local study examining the profile of methamphetamine-related admissions to specialist psychiatric hospitals in Cape Town also showed that most patients were male, had an average age of 25 years, and were diagnosed with a substance-induced psychosis (41\%) followed by schizophrenia (31\%). ${ }^{[13]}$ Methamphetamine use has consistently been found to be associated with several deleterious mental health outcomes, including depression, suicidal ideation and psychosis. ${ }^{[14,15]}$ This drug is also the primary substance of abuse in the Western Cape (38\%), and is mainly abused by men, with an average age of 26 years. ${ }^{[3]}$ Furthermore, the Helderberg subdistrict has previously been found to have higher rates of lifetime, weekly and daily methamphetamine use than other districts of the Cape Town Metropole. ${ }^{[16]}$ In summary, the Western Cape has emerged as one of the regions in the world with the highest levels of methamphetamine use, ${ }^{[17]}$ which places an enormous burden on healthcare services, and more specifically, mental healthcare.

Given the high prevalence of substanceinduced psychosis (53\%) and the secondary diagnostic coding for these admissions, care users. A descriptive study completed in it appears that these patients had a 'dual diagnosis', denoting the co-occurrence of substance abuse and mental illness. Comorbidity of mental illness with substance abuse is especially relevant in relation to treatment and prognostic issues, while the negative effect on the overall health of a patient adds an additional burden to service delivery. ${ }^{[18]}$ Dual diagnoses patients are also not only admitted at entry-level care at Helderberg, but also at level-2 care. A prospective, descriptive prevalence study at a level-2 hospital in the Western Cape, i.e. Stikland, showed that $51 \%$ of patients were diagnosed with comorbid substance use (abuse or dependence). ${ }^{[18]}$ Although we cannot rule out that the high proportion of admissions for substance misuse may be related to a coding issue, the data point to a potentially very high inpatient resource use for substance-related problems.

Primary or secondary substance use disorders further accounted for $61 \%$ of abscondments and 57\% of readmissions, of which $65 \%$ occurred within 3 months of discharge. These findings suggest that a high proportion of patients were difficult to manage, further emphasising the need for service planning in terms of designated psychiatric beds and seclusion rooms. A review by Ramlall ${ }^{[19]}$ on the longitudinal effect of the MHCA on mental healthcare service delivery accordingly states that one of the most common complaints was managing aggressive and behaviourally disturbed patients in a general hospital setting in the absence of seclusion rooms.

While a major responsibility of district hospitals in terms of the MHCA is the provision of short-term inpatient care for a period of 72 hours prior to transfer to the next level of care, the legislation has proven difficult to implement in practice. This has resulted in patients who require further involuntary admission and care staying longer at entry level, with additional pressures on districtlevel hospital resources.

In terms of outcomes, only $18 \%$ of admission episodes were transferred to specialist/tertiary psychiatric hospitals, while $76 \%$ were discharged into the community without being transferred to tertiary level for further management. This is in accordance with the objectives as set out in the MHCA, which promotes a decentralised model of care. In addition, a factor to consider with 
respect to the purpose of the 72-hour observation assessment period is the exclusion of a medical cause for altered mental state or behavioral disturbance. In this sample, only $5 \%$ of mental healthcare users were transferred to medical or surgical services. In contrast, the low number of admission episodes transferred to specialist/tertiary care could be ascribed to the limited availability of beds at this level of care. Lund et al. ${ }^{[20]}$ reported a $7.7 \%$ reduction from 2000 to 2005 in the availability of hospital beds at a tertiary level across all provinces. In addition, secondary-level hospitals are required to provide psychiatric care and treatment to special population groups, such as children, adolescents and the elderly, in the absence of dedicated psychiatric beds. Hence, patients from level 1 may not be transferred to specialist/ tertiary level psychiatric care following the 72-hour assessment period.

There was also a significant difference in queuing time between the specialist/tertiary psychiatric hospitals, which could be ascribed to, inter alia, the demographic profile of patients, the limited number of beds available at specialist/tertiary psychiatric hospitals for the patient profiles admissible to these centres, and type of care and treatment provided for special patient population groups (child and adolescent, geriatric and intellectual disability).

\section{Limitations and strengths}

While the data comprise a complete and validated set from only one health centre, care should be taken to extrapolate the results of this study to guide service planning of other district-level hospitals, as the population of patients admitted to Helderberg Hospital may not be representative of those admitted to other district-level hospitals. Furthermore, while clinical diagnoses were made in accordance with the DSM-IV diagnostic system, these were not verified in all instances prior to discharge or transfer.

\section{Conclusions}

This study, although limited in scope, suggests that substance abuse, specifically of methamphetamine, places a significant burden on mental healthcare services at entry level. It is further noteworthy that only $18 \%$ were transferred to specialist/tertiary psychiatric hospitals, while $76 \%$ of admission episodes who received treatment were discharged into the community, in accordance with the objectives set out in the MHCA, which promotes a decentralised model. However, in order to translate legislation as stipulated in the MHCA into reality with regards to the care of MHCUs at district hospitals, emphasis needs to be placed on improving infrastructure and resources. The high abscondment rates and length of stay extending beyond the prescribed 72-hour assessment period at this facility highlight the issue of infrastructure and resource constraints, albeit at this facility alone. We recommend the provision of adequate seclusion rooms, as well as designated beds available for the care of MHCUs rather than management in a general ward setting. Furthermore, given the high incidence of substance-induced and substance use disorders and their effect on outcomes such as readmission rates, we would recommend that service planning should further include mental health and substance abuse intervention systems as well as training to better manage substance use disorders and dual diagnosis. Finally, discharge planning should extend into community substance abuse education, while patients should be linked with posthospital rehabilitative treatment services and community support structures.

Acknowledgments. The authors hereby acknowledge Dr Lize Weich and Prof. Liezl Koen for providing helpful suggestions to improve the manuscript. We would also like to thank Sr Charlene Lundall-Stoltz and Dr Michelle van Zyl for their assistance with the initial collection of data.

\section{References}

1. Herman A, Stein DJ, Seedat S, Heeringa SG, Moomal H, Williams DR. The South African Stress and Health (SASH) study: 12-month and lifetime prevalence of common mental disorders. S Afr Med J 2009;99(5 Pt 2):339-344.

2. Kleintjies S, Flisher AJ, Railoun A, Lund C, Moltano C, Robertson BA. The prevalence of mental disorders among children, adolescents, and adults in the Western Cape, South Africa. S Afr Psychiatry Rev 2006;9(3):157-160.

3. Dada S, Plüddemann A, Parry C, et al. Monitoring alcohol and drug abuse trends in South Africa (July 1996 - December 2011). SACENDU Research Brief 2012;15(1):1-14.

4. Vos PJ, Cloete KJ, le Roux A, Kidd M, Jordaan GP. A retrospective review of trends and clinical characteristics of methamphetamine-related acute psychiatric admissions in a South African context. Afr J Psychiatry 2010;13(5):390-394.

5. Mental Health Care Act No. 17. Government Gazette 2005, 464 (27300). Pretoria: Government Printing Works, 2002.

6. Kaliski S. Psycho-legal Assessment in South Africa. Cape Town: Oxford University Press, 2006.

7. Ramlall S, Chipps J, Mars M. Impact of the South African Mental Health Care Act No. 17 of 2002 on regional and district hospitals designated for mental health care in KwaZulu-Natal. S Afr Med J 2010;100(10):667-670.

8. Burns JK. Implementation of the Mental Health Care Act (2002) at district hospitals in South Africa: Translating principles into practice. S Afr Med J 2008;98(1):46-49.

9. Petersen I, Lund C. Mental health service delivery in South Africa from 2000 to 2010: One step forward, one step back. S Afr Med J 2011;101(10):751-757.

10. American Psychiatric Association. Diagnostic and Statistical Manual of Mental Disorders. 4th edition (DSM-IV). Washington DC: American Psychiatric Association, 1994.

11. World Medical Association. Declaration of Helsinki: Ethical Principles for Medical Research Involving Human Subjects. Finland: World Medical Association, 2000.

12. Habib T, Van Rooyen FC, Hiemstra LA. Involuntary admission of psychiatric patients in the Northern Cape Province and the accuracy of the initial psychiatric assessment done by the referring general practitioners. S Afr Fam Pract 2007;49(6):14a-14c. [http://dx.doi. org/10.1080/20786204.2007.10873566]

13. Plüddemann A, Dada S, Parry CDH, et al. Monitoring the prevalence of methamphetaminerelated presentations at psychiatric hospitals in Cape Town, South Africa. Afr J Psychiatry 2013;16(1):45-49. [http://dx.doi.org/10.4314/ajpsy.v16i1.8.]

14. Marshall BD, Werb D. Health outcomes associated with methamphetamine use among young people: A systematic review. Addiction 2010;105(6):991-1002. [http://dx.doi.org/10.1111/ j.1360-0443.2010.02932.x]

15. Plüddemann A, Flisher AJ, McKetin R, Parry C, Lombard C. Methamphetamine use, aggressive behavior and other mental health issues among high school students in Cape Town, South Africa. Drug Alcohol Depend 2010;109(1-3):14-19. [http://dx.doi.org/10.1016/j. drugalcdep.2009.11.021]

16. Harker N, Kader R, Myers B, et al. Substance Abuse Trends in the Western Cape: A Review of Studies Conducted Since 2000. Parow: Medical Research Council, 2008.

17. United Nations Office on Drug and Crime. World Drug Report. New York: United Nations Publications, 2011.

18. Weich L, Pienaar W. Occurrence of comorbid substance use disorders among acute psychiatric inpatients at Stikland Hospital in Western Cape, South Africa. Afr J Psychiatry 2009;12(3):213-217.

19. Ramlall S. The Mental Health Care Act No. 17 - South Africa, Trials and triumphs. Afr J Psychiatry 2012;15(6):407-410. [http://dx.doi.org/10.4314/ajpsy.v15i6.49]

20. Lund C, Kleintjies S, Kakuma R, Fisher AJ; MhaPP Research Programme Consortium. Public sector mental health systems in South Africa: Inter-provincial comparisons and policy implications. Soc Psychiatry Psychiatr Epidemiol 2010;45(3):393-404. [http://dx.doi. org/10.1007/s00127-009-0078-5] 